\title{
The Effect of Probiotics on Prevention of Common Cold: A Meta-Analysis of Randomized Controlled Trial Studies
}

\section{En-Jin Kang, Soo Young Kim*, In-Hong Hwang, Yun-Jeong Ji}

Department of Family Medicine, Kangdong Sacred Heart Hospital, Hallym University College of Medicine, Seoul, Korea

Background: Probiotics are currently under focus for their immune improvement function. Many studies have been performed to assess the potential efficacy of probiotics in allergic disease, viral disease, respiratory disease, as well as gastrointestinal disease. This study performed a systematic review to determine the effects of probiotics on the prevention of the common cold.

Methods: We searched MEDLINE (PubMed), EMBASE, CINAHL, and Cochrane CENTRAL for studies released through June 2011. Two authors independently extracted the data. To assess the risk of bias of included literatures, Cochrane Collaboration's risk of bias tool was used.

Results: We identified 10 studies in 7 articles. A total 2,894 participants, 1,588 in the probiotics group and 1,306 in the control group, were included. The effect of probiotics on the prevention of the common cold had a relative risk (RR) of 0.92 (95\% CI, 0.85 to $1.00, \mathrm{I}^{2}=26 \%$ ). In the subgroup analysis, the RR of administration of probiotics for 3 months or less was 0.82 (95\% CI, 0.70 to 0.97 ). The RR of administration of probiotics over 3 months was 1.00 (95\% CI, 0.92 to 1.09 ). The RR of administration of probiotics without any active intervention (vitamin and mineral) was 0.87 (95\% CI, 0.78 to 0.97 ).

Conclusion: In this meta-analysis, there was marginal effect of probiotics on the prevention of the common cold. The results implied that probiotics had a modest effect in common cold reduction. The balance of benefit and harms needs to be considered when using probiotics for common cold prevention.

Keywords: Probiotics; Common Cold; Meta-Analysis

\section{INTRODUCTION}

Received: August 25, 2011, Accepted: October 12, 2012

${ }^{*}$ Corresponding Author: Soo Young Kim

Tel: +82-2-2224-2406, Fax: +82-2-2224-2409

E-mail: hallymfm@gmail.com

Korean Journal of Family Medicine

Copyright (C) 2013 The Korean Academy of Family Medicine

(ㄷ) This is an open-access article distributed under the terms of the Creative Commons Attribution Non-Commercial License (http://creativecommons.org/licenses/by-nc/3.0) which permits unrestricted noncommercial use, distribution, and reproduction in any medium, provided the original work is properly cited.

The common cold is an upper respiratory tract infection and is one of the most common diseases occurring in all ages. Since the common cold cannot be cured, the best thing to do is to avoid catching the virus that causes the common cold. There have been many randomized controlled studies investigating whether taking vitamin $\mathrm{C}$, garlic, or echinacea has an effect on preventing colds. According to a systematic review, there was no significant effect from these supplements in most cases. ${ }^{1-3)}$ 
Probiotics can be defined as "living microorganisms that confer a health benefit when consumed in adequate amount." ${ }^{\text {"4) }}$ Probiotics are used as dietary supplements or remedies for a variety of gastrointestinal disorders. Recently, it was discovered that probiotics may improve immune function. For this reason, there has been increased interest in prophylactic daily use of probiotics. According to the Cochrane review, the prophylactic use of probiotics reduces the incidence of necrotic enterocolitis (NEC) and risk of NEC related death in comparison to the control group, ${ }^{5)}$ and probiotics lower the risk for antibiotic-associated diarrhea in children, ${ }^{6)}$ Trials have been conducted on the efficacy of probiotics against a wide range of diseases, including allergic diseases, viral diseases, respiratory diseases, cancer, and digestive diseases.

Probiotics may have a positive preventative effect against the common cold because the cold is a viral disease. Some studies revealed that probiotics have preventive effect against colds, but there has been no systematic review of this subject. However, based on a systematic review targeting 14 studies on the preventive effect of probiotics against respiratory disease, four studies ${ }^{7)}$ reported that the incidence of respiratory disease was reduced after taking probiotics and ten studies reported that there was no reduction. The purpose of this study is to investigate whether there is a cold-preventative effect of probiotic administration through a systematic review of the literature of randomized controlled trials.

\section{METHODS}

A systematic review of the literature for randomized controlled trials was performed to see whether there is a coldpreventative effect of probiotic administration in the general population.

\section{Inclusion Criteria}

Randomized controlled trials were selected that compare the difference in the incidence of colds and cold symptoms, or rhinopharyngitis, between a study group with probiotics and a control group without probiotics or probiotic-containing drinks (placebo or non-treatment). Studies about upper respiratory tract diseases other than the common cold, such as sinusitis, tonsillitis, laryngitis, otitis media; studies about the prevention of lower respiratory tract disease, such as bronchitis, pneumonia; studies including a group likely to be influenced by other active medication, such as vitamine; and studies about the relief or improvement of cold symptoms were excluded.

\section{Search Method}

The last search was performed in June 2011. All searches were made by professional librarians and search terms were selected in consultation between the authors and professional librarians. There were no restrictions on the language of the papers published. Databases searched include: MEDLINE (PubMed), EMBASE, Cochrane Central Register of Controlled Trials (CENTRAL), and CINAHL. The following search terms were used.

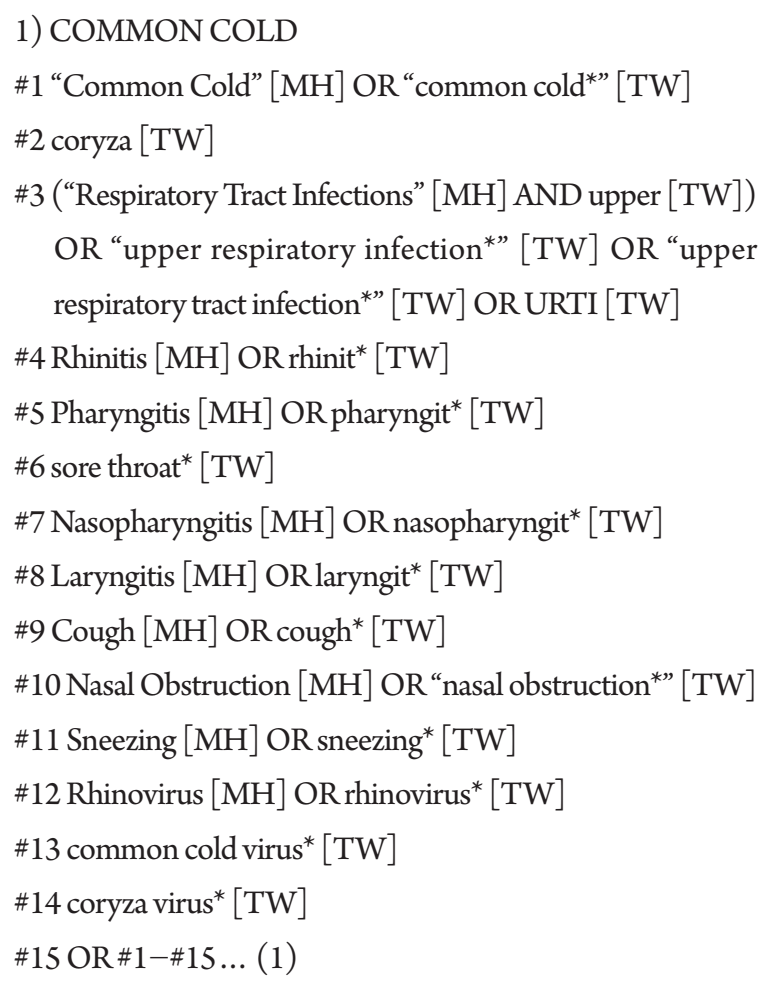

\section{2) PROBIOTICS}

\#1 Probiotics [MH] OR probiotics [TW]

\#2 Lactobacillus [MH] OR lactobacill* [TW]

\#3 Bifidobacterium [MH] OR bifidobact* [TW]

\#4 Lactococcus [MH] OR lactococc* [TW]

\#5 Enterococcus $[\mathrm{MH}]$ OR enterococc* [TW]

\#6 OR\#1-\#5 ... (2) 
3) Randomized controlled trial

\#1 randomized controlled trial [PT]

\#2 controlled clinical trial [PT]

\#3 randomized [TW]

\#4 placebo [TW]

\#5 drug therapy [subheading]

\#6 randomly [TW]

\#7 trial [TW]

\#8 groups [TW]

\#9 OR\#1-\#8... (3)

animals $[\mathrm{mh}]$ NOT humans $[\mathrm{mh}] \ldots$ (4)

[(1) AND (2) AND (3)] NOT (4)

\section{Study Selection}

Two independent authors reviewed the results of the searches and the papers that met the inclusion criteria were selected. In the case of disagreement, the paper was reviewed again and the decision was made by discussion and consensus. If consensus was not possible, a final decision was made by the third author.

\section{Assessment of Risk of Bias}

The quality of studies for selected research was performed by using the Cochrane Collaboration's tool for assessing risk of bias. ${ }^{8)}$ Each criteria was assessed as one of: yes, no, and unclear, with 'yes' indicating a low risk of bias, 'no' indicating a high risk of bias, and 'unclear' indicating a lack of information. The evaluation was done by two independent authors, and discrepancies were resolved by discussion and consensus.

\section{Data Extraction}

Relevant data, such as the study design, subject and characteristics, type and amount of probiotics and intake method, control group, the follow-up period, and outcome variables were abstracted independently by two review authors. Discrepancies were resolved by discussion and consensus.

\section{Statistical Analysis}

The 95\% confidence intervals (CI) for the relative risk are presented as follows. Review Manager ver. 5.1 (RevMan) ${ }^{9)}$

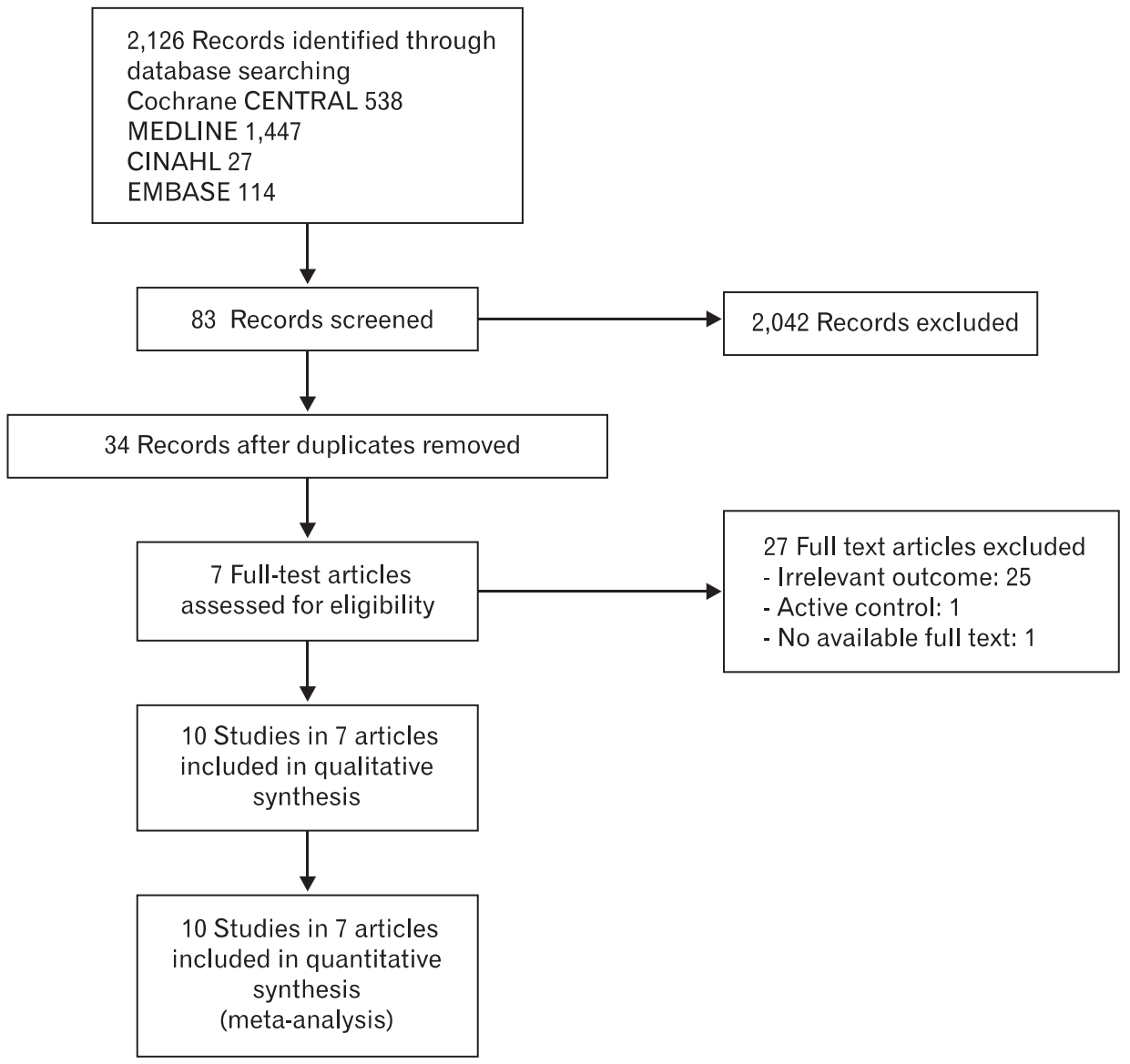

Figure 1. Flow sheet of study selection. 


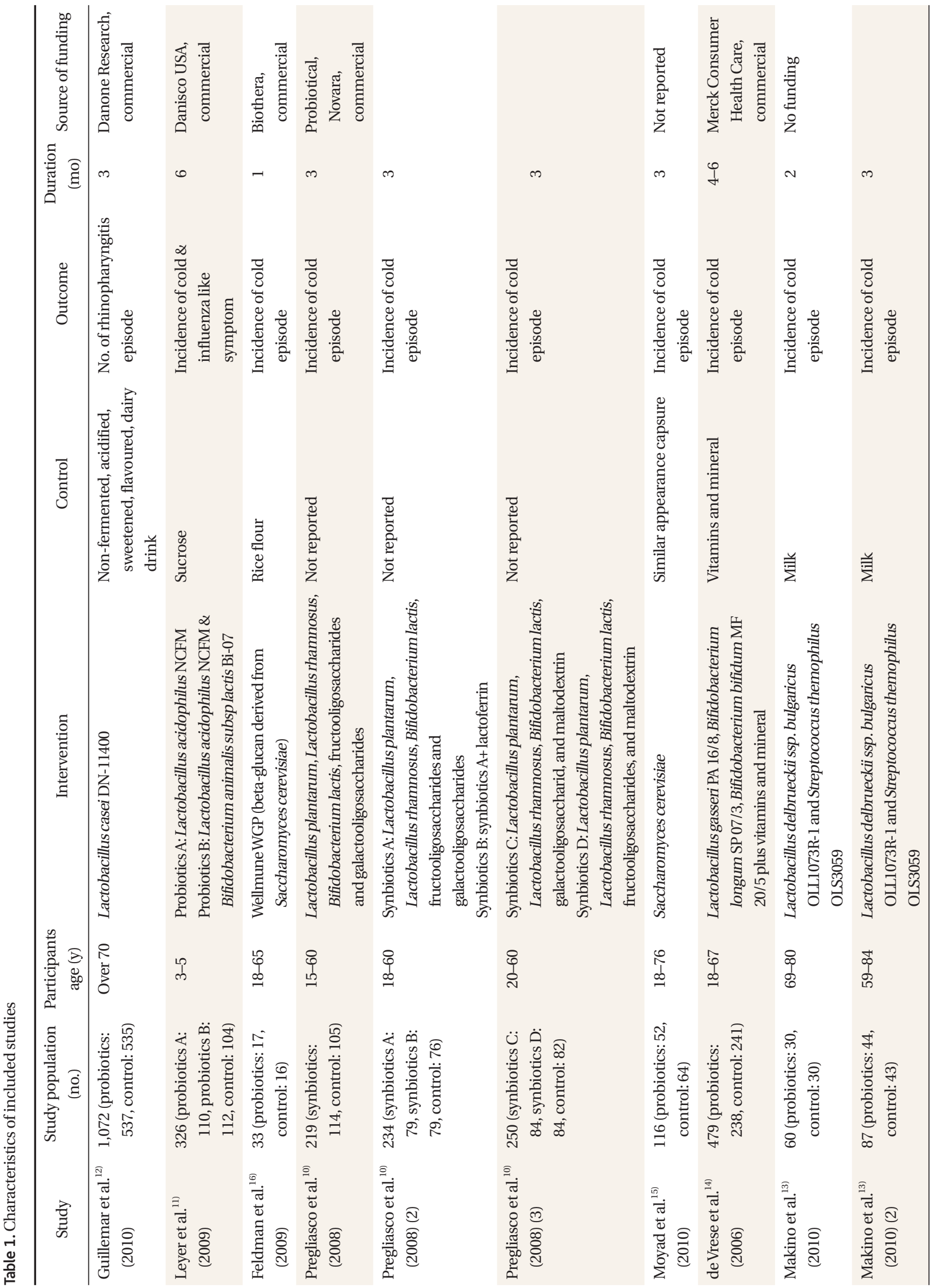


software was used for statistical analysis. As a data analysis method, a fixed effect model was applied when the homogeneity between studies was verified. The results were displayed in a forest plot. An $\mathrm{I}^{2}$ test was applied to determine between-study heterogeneity. A subgroup analysis was conducted to investigate age, probiotics treatment duration, and treatment variables. A funnel plot was designed to check the existence of publication bias.

\section{RESULTS}

Based on the electronic search, 538 abstracts from Cochrane, 1,447 from MEDLINE, 144 from EMBASE, and 29 from CINAHL, for a total of 2,126 abstracts were found. Out of these, a total of seven randomized controlled trials were selected and included in the analysis (Figure 1). ${ }^{10-16)}$ The characteristics of the included studies are shown in Table 1.

A total of 2,894 participants were included: 1,580 participants were in the probiotic group and 1,296 were in the control group. The randomized controlled trials ${ }^{13)}$ were analyzed as two separate studies, and another study consisting of three randomized controlled trials was included in the analysis individually as well. The number of subjects in these studies was selected by the intention-to treat (ITT) principle, with the exception of one study ${ }^{16)}$ which used a modified ITT analysis including only subjects who received at least one dose of the study medication.
If results of a study were only described by odds ratios ${ }^{12)}$ instead of the number of patients, then the number of patients was calculated and analyzed using the available information. The risk of bias for the included studies was evaluated by using Cochrane Collaboration's tool for assessing risk of bias. ${ }^{5)}$ It was evaluated that items for appropriate random sequence generation were performed properly in four studies. ${ }^{10,12,14,16)}$ Allocation concealment was adequately described in five studies ${ }^{11,12,14-16)}$ but in one study, milk was used instead of yogurt as a control, which was considered to be inadequate. Blinding of intervention and outcome was adequately described except in one study. ${ }^{13)}$ Incomplete outcome data were adequately addressed in all studies. Also, in one study ${ }^{11)}$ the ages of the control group were much higher than the probiotic groups, and in another study ${ }^{15)}$ it was not clear whether or not the study participants received a flu vaccine. There was a possibility of potential bias in these two studies. In addition, one study ${ }^{14)}$ which used vitamins and minerals both in the probiotics group and in the control group was assessed to be at a high risk of bias. All except one study ${ }^{13)}$ were assessed as relatively higher quality studies (Figure 2 ). The investigators of five studies ${ }^{10-12,14,16)}$ out of the seven were funded by a commercial company. The funding of one study ${ }^{15)}$ was not disclosed. Only one study ${ }^{13)}$ was done without any funding. Based on ten studies extracted from seven papers, the relative risk of the common cold in probiotics groups compared to the control group was 0.92 (95\% CI, 0.84 to $1.00 ; \mathrm{I}^{2}=27 \%$ ) (Figure 3).

Using the results of seven papers encompassing a total of

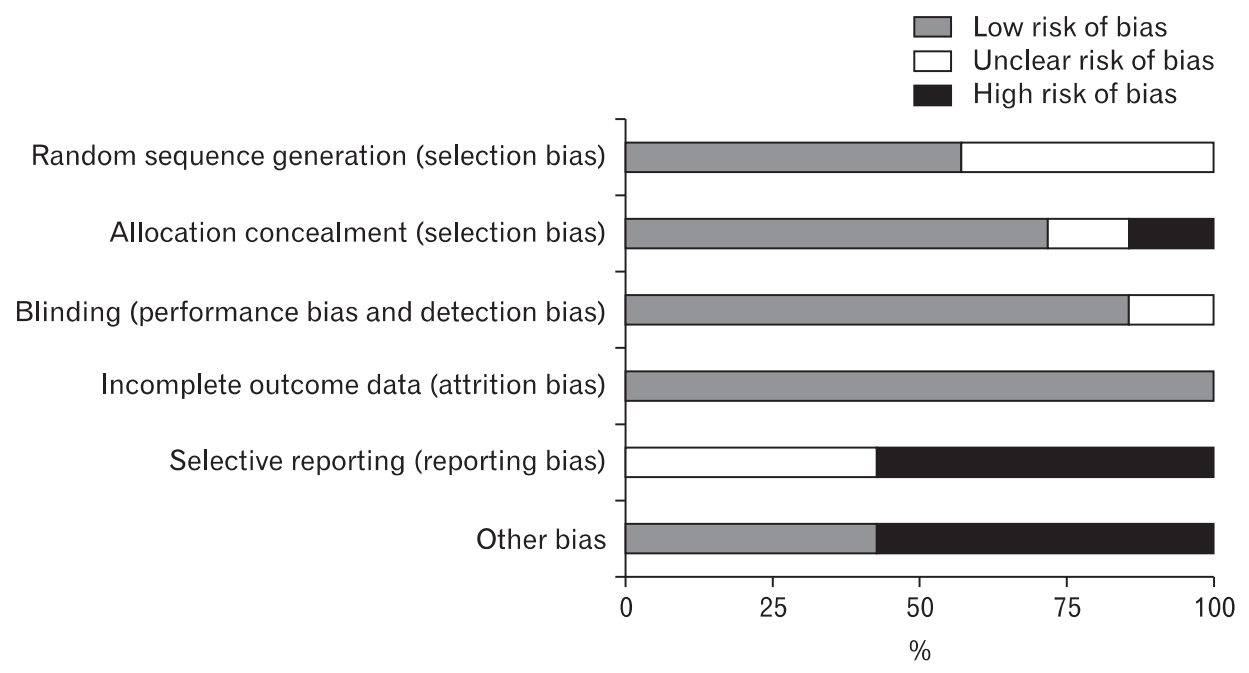

Figure 2. Risk of bias graph. 


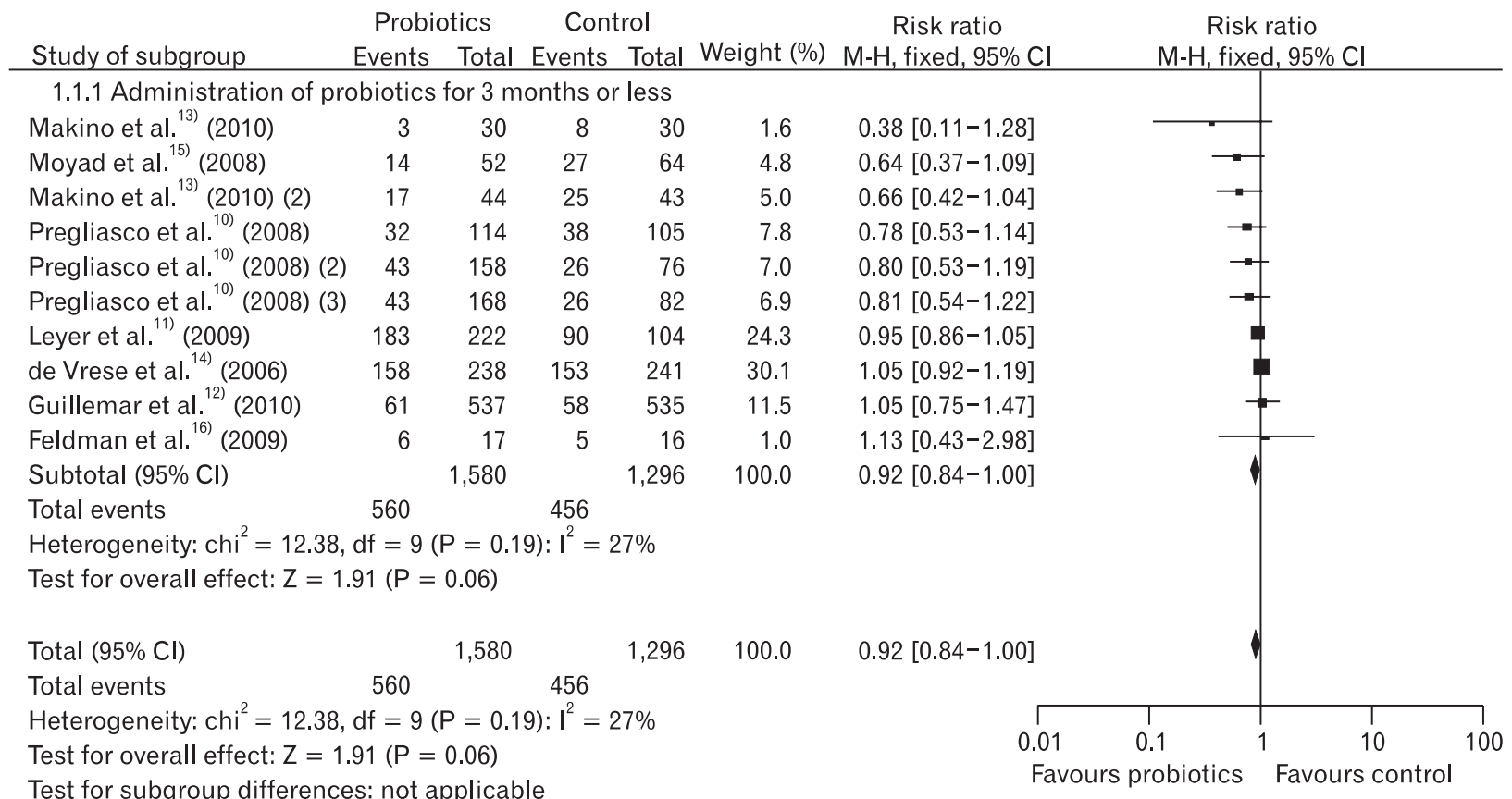

Figure 3. Meta-analysis of studies probiotics in common cold prevention.

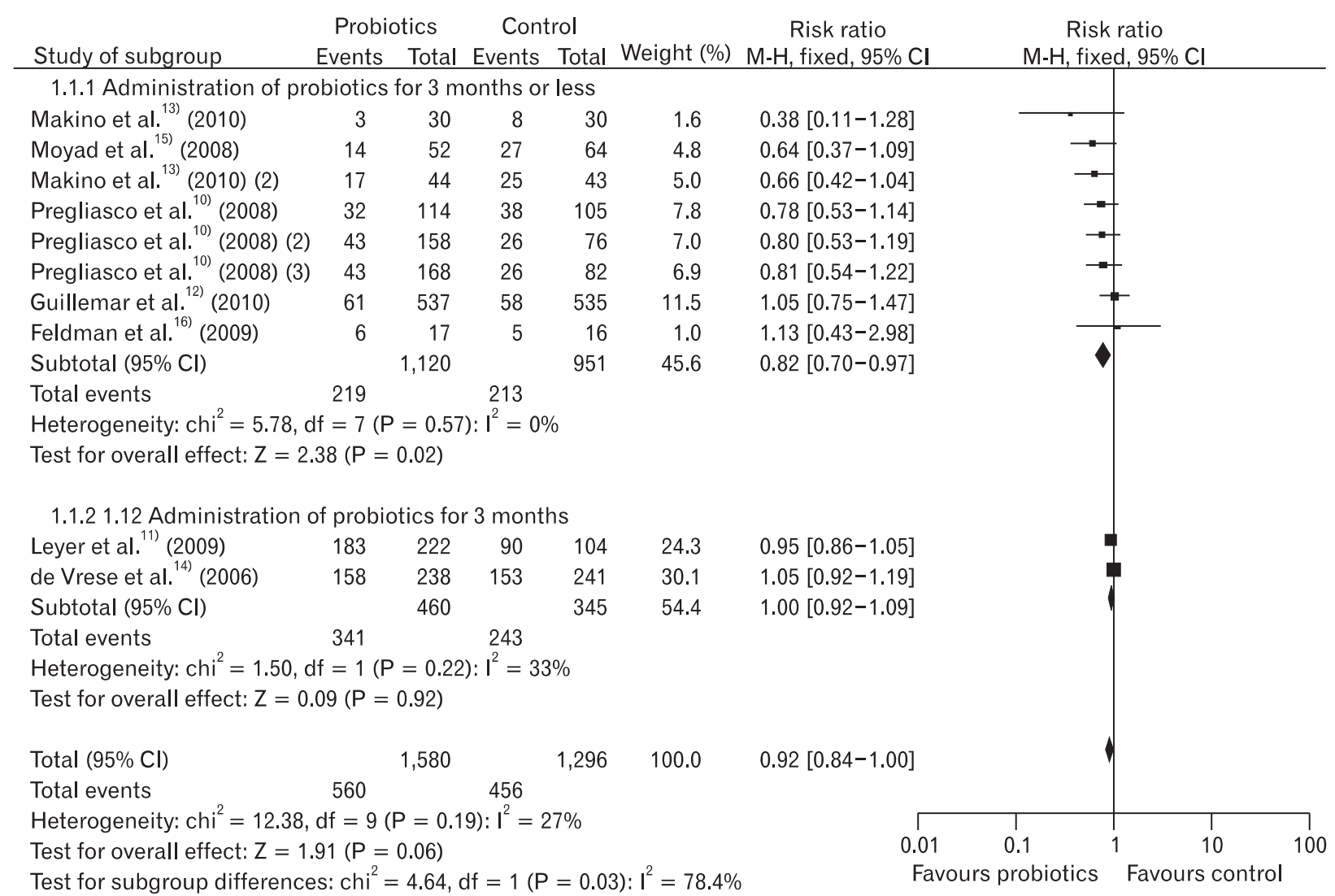

Figure 4. Subgroup analysis: duration of probiotics administration. 


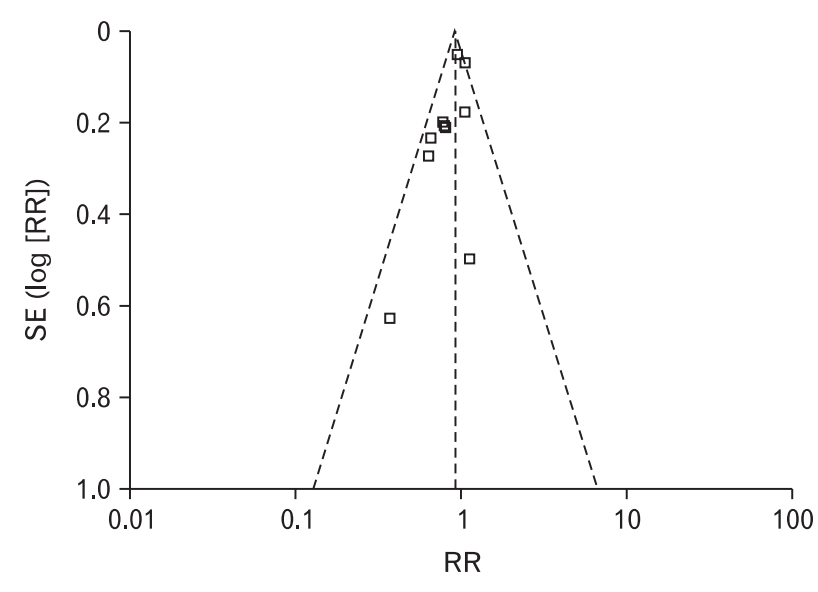

Figure 5. Funnel plot. RR: relative risk.

ten studies, subgroup analysis was performed based on study characteristics. First, a subgroup analysis was done based on the probiotics administration period. When the probiotics administration period was less than three months, the relative risk was 0.82 ( $95 \% \mathrm{CI}, 0.70$ to $0.97 ; \mathrm{I}^{2}=0 \%$ ), and when it was longer than three months, the relative risk was 1.00 (95\% CI, 0.92 to 1.09; $\mathrm{I}^{2}=33 \%$ ) (Figure 4). Based on the result of the comparison between probiotic administration with vitamins and minerals and just probiotic administration, the relative risk excluding one study $^{14)}$ was 0.87 (95\% CI, 0.78 to $0.97 ; \mathrm{I}^{2}=22 \%$ ). Excluding a pediatric research study, ${ }^{11)}$ the relative risk was 0.91 (95\% CI, 0.82 to $\left.1.021 ; I^{2}=34 \%\right)$. Excluding one low quality study, ${ }^{13)}$ the relative risk was 0.93 (95\% CI, 0.85 to $1.01 ; \mathrm{I}^{2}=19 \%$ ). Excluding two studies ${ }^{10,13)}$ in which it is uncertain whether a placebo was used, the relative risk was 0.97 (95\% CI, 0.88 to 1.06 ; $\mathrm{I}^{2}=26 \%$ ). A subgroup analysis in relation to gender, types of probiotics and daily dose, and underlying disease was not performed since each study was very different or because these factors were not properly reported in the papers.

None of the included studies reported significant differences in adverse effects between the two groups in relation to changes in bowel movements, musculoskeletal symptoms, gastrointestinal symptoms, infection, or neurological or psychiatric symptoms. Any such effects were not severe. A distinct asymmetry in the Funnel plot was not observed (Figure 5).

\section{DISCUSSION}

Cold is generally a mild illness and is usually overcome naturally. Therefore, in order to be clinically useful, a cold prevention method should be simple, low cost, and without adverse reactions. In this systematic review of whether there is a cold-preventative effect in probiotic administration, there was no statistically significant evidence overall, but probiotics seemed to have borderline preventive effects. The preventive effect was mainly observable with probiotics administration periods of less than three months.

Despite a comprehensive search, only ten studies met the inclusion criteria. Studies about upper respiratory tract diseases other than the common cold and studies including a group likely to be influenced by other active medication, such as vitamins were excluded. Generally, the overall quality of studies was relatively high.

In summary, there is the possibility that probiotics have a cold preventive effect, but such a preventive effect is not conclusively demonstrated. Since there were no previous systematic reviews concerning the use of probiotics for the prevention of the common cold, comparison of results was not possible.

Therefore, when taking probiotics constantly, it is difficult to exclude the possibility of a somewhat lower risk of developing a cold. There are no reported serious side effects of the use of probiotics in the current literature. However, the use of probiotic administration for cold prevention must be determined by considering the severity of colds, and the cost and inconvenience of administering probiotics.

A subgroup analysis was performed based on the length of the probiotics administration period. When the period was less than three months, the occurrence of the common cold decreased by $18 \%$, but if it was longer than three months, it had no effect on cold prevention. Typically, more people suffer from colds during a change in season. ${ }^{17)}$ Therefore after three months, the probability of the occurrence of a common cold can be increased, which may be an explanation of our results. It should be considered whether medication period occurred during a change in season; without doing so it is hard to make the conclusion that short-term administration of probiotics has a preventive effect.

Based on the other subgroup analysis, results of the 
comparison between administration of probiotics with vitamins and minerals and just probiotic administration showed that incidence of the common cold decreased by $13 \%$. The coldpreventive effects of probiotics may have been diminished due to the use of vitamins, but it is difficult to draw any clear conclusions because only one study was analyzed. Whether cold prevention effects might be different between adults and young children is hard to determine because there was only one pediatric study investigated. In addition, subgroup analyses concerning gender, types of probiotics, daily dose, and underlying disease were not performed since each study is very different or such factors were not properly reported in the papers.

This study has several limitations: first, since the common cold cannot be diagnosed from clinical symptoms alone and objective diagnostic indicators for the common cold do not exist, occurrence of the common cold can be missed or misdiagnosed. This can influence the outcome of the research. Four studies included in this systematic review chose to diagnose based on clinical symptoms determined by questionnaires. However, these methods do not distinguish clearly between the common cold and other respiratory diseases including lower respiratory diseases. It may be necessary to conduct a large scale study in which experts differentiate colds from other respiratory infections. Second, differences in cold prevention effects can exist depending on the kinds and the amount of probiotics used. There are differences between probiotic strains in their ability to clean the intestine, and the there is evidence that viability of Streptococcus themophilus and Lactobacillus delbrueckii ssp. bulgaricus in the intestine is weak. ${ }^{18,19)}$ Since each type of probiotic might have a different effect on cold prevention, future research on effectiveness will be required taking into account the type and number of strains, methods of mixing strains, and process.

In conclusion, the cold preventive effects of probiotics administration was investigated through a systematic review of randomized controlled trials, and the results showed that there was no statistically significant cold prevention overall, but there may be a borderline preventive effect. A preventive effect was found mainly in the case of a study period of less than three months and it appeared when a placebo group was used as the control group. However, the decision as to whether to take a probiotic for prevention of the common cold should be made by considering the severity of the cold and the cost and inconvenience of probiotics administration.

\section{CONFLICT OF INTEREST}

No potential conflict of interest relevant to this article was reported.

\section{REFERENCES}

1. Linde K, Barrett B, Wolkart K, Bauer R, Melchart D. Echinacea for preventing and treating the common cold. Cochrane Database Syst Rev 2006; (1):CD000530.

2. Douglas RM, Hemila H, Chalker E, Treacy B. Vitamin C for preventing and treating the common cold. Cochrane Database Syst Rev 2007; (3):CD000980.

3. Lissiman E, Bhasale AL, Cohen M. Garlic for the common cold. Cochrane Database Syst Rev 2009; (3):CD006206.

4. Reid G, Jass J, Sebulsky MT, McCormick JK. Potential uses of probiotics in clinical practice. Clin Microbiol Rev 2003;16: 658-72.

5. Alfaleh K, Anabrees J, Bassler D, Al-Kharfi T. Probiotics for prevention of necrotizing enterocolitis in preterm infants. Cochrane Database Syst Rev 2011;(3):CD005496.

6. Johnston BC, Supina AL, Ospina M, Vohra S. Probiotics for the prevention of pediatric antibiotic-associated diarrhea. Cochrane Database Syst Rev 2007; (2):CD004827.

7. Vouloumanou EK, Makris GC, Karageorgopoulos DE, Falagas ME. Probiotics for the prevention of respiratory tract infections: a systematic review. Int J Antimicrob Agents 2009; 34:197.e1-10.

8. Higgins JP, Altman DG. Assessing risk of bias in included studies. In: Higgins JP, Green S, editors. Cochrane handbook for systematic reviews of interventions: version 5.0.0 [Internet]. Chichester: Wiley-Blackwell; 2008 [cited 2011 Aug 1]. Available from: http://www.cochrane-handbook.org. 9. Review Manager (RevMan) [Computer program]. ver. 5.0. Copenhagen: The Nordic Cochrane Centre, The Cochrane Collaboration; 2011.

10. Pregliasco F, Anselmi G, Fonte L, Giussani F, Schieppati $S$, Soletti L. A new chance of preventing winter diseases 
by the administration of symbiotic formulations. J Clin Gastroenterol 2008;42 Suppl 3 Pt 2:S224-33.

11. Leyer GJ, Li S, Mubasher ME, Reifer C, Ouwehand AC. Probiotic effects on cold and influenza-like symptom incidence and duration in children. Pediatrics 2009;124:e1729.

12. Guillemard E, Tondu F, Lacoin F, Schrezenmeir J. Consumption of a fermented dairy product containing the probiotic Lactobacillus casei DN-114001 reduces the duration of respiratory infections in the elderly in a randomised controlled trial. Br J Nutr 2010;103:58-68.

13. Makino S, Ikegami S, Kume A, Horiuchi H, Sasaki H, Orii N. Reducing the risk of infection in the elderly by dietary intake of yoghurt fermented with Lactobacillus delbrueckii ssp. bulgaricus OLL1073R-1. Br J Nutr 2010;104:998-1006.

14. de Vrese M, Winkler P, Rautenberg P, Harder T, Noah C, Laue C, et al. Effect of Lactobacillus gasseri PA 16/8,
Bifidobacterium longum SP 07/3, B. bifidum MF 20/5 on common cold episodes: a double blind, randomized, controlled trial. Clin Nutr 2005;24:481-91.

15. Moyad MA, Robinson LE, Zawada ET Jr, Kittelsrud JM, Chen DG, Reeves SG, et al. Effects of a modified yeast supplement on cold/flu symptoms. Urol Nurs 2008;28:50-5.

16. Feldman S, Schwartz HI, Kalman DS, Mayers A, Kohrman HM, Clemens R, et al. Randomized phase II clinical trials of Wellmune WGP for immune support during cold and flu season. J Appl Res 2009;9:30-42.

17. Heikkinen T, Jarvinen A. The common cold. Lancet 2003; 361:51-9.

18. Kim J, Han S. The clinical effect and use of probiotics. J Korean Acad Fam Med 2008;29:467-74.

19. Kim NJ, Ji GE. Probiotics: action mechanisms and clinical effects. Pediatr Allergy Respir Dis 2005;15:327-43. 